\title{
Remote supervision strategy based on in-use vehicle OBD data flow
}

\author{
Tiantian Wang ${ }^{1, *}$, Junjie Liu ${ }^{1}$, Chuan $\mathrm{Wan}^{2}$, and Zhi $\mathrm{Wang}^{3}$ \\ ${ }^{1}$ School of mechanical and electrical engineering and vehicle engineering, East China Jiaotong \\ University, Nanchang 330100, China \\ ${ }^{2}$ Power transmission development department, Jiangling Automobile Co., Ltd., Nanchang 330100, \\ China \\ ${ }^{3}$ AVL List Technical Center (Shanghai) Co., Ltd., Shanghai 201206, China
}

Keywords: OBD, remote monitoring, platform, diagnosis, emission.

\begin{abstract}
Test results from many researchers show that $\mathrm{NO}_{x}$ emissions from parts of on-road heavy-duty diesels are higher than which been registered. Therefore, CN VI emission regulations clearly proposes that the heavy-duty diesels should be supervised by a T-BOX which can transmit CAN message from vehicle OBD interface to the remote monitoring platform. Recognition of cheating behaver and calculation method of $\mathrm{NO}_{x}$ emission were developed and verified by platform data from on-line vehicle. The modules in the platform can effectively monitor urea consumption rationality, injection system working state, exhaust temperature sensor signal reasonability, $\mathrm{NO}_{x}$ sensor signal reasonability and $\mathrm{NO}_{x}$ emission level, which can reduce the cost of supervision and governance, improve the effectiveness of regulation and provide basic data for policy making.
\end{abstract}

\section{Introduction}

There is a long time since domestic research on vehicle remote monitoring platform have been started. For example, the research of Ding Jinquan, Qiu Yuan, Wang Yu and Liao Yanhui ${ }^{[1-4]}$ mainly aims at fleet management, vehicle safety, traffic violations, OBD fault alarm information upload, etc. But the information uploaded is limited, which cannot collect vehicle OBD interface data in depth, and limits the potential of platform data mining; In addition, He Penglin ${ }^{[5]}$ et al. set up a warning and monitoring platform for excessive emission of automobile exhaust in Shenzhen using OBDII auto-diagnosis and wireless communication technology; Dong Xiaoling ${ }^{[6]}$ of Beijing University of Technology established a modular and extensible framework of automobile remote diagnosis and status monitoring system by using the spatial separation method of TRIZ theory, and built a software system of remote diagnosis based on OBD device, mobile APP and remote server; Yan Xupu ${ }^{[7]}$ of Beijing University of Technology has established a monitoring platform with preliminary functions for the design of a remote monitoring system for vehicle exhaust emissions based on OBD data. These researches mainly focus on data collection methods, data upload methods, data management,

\footnotetext{
*Corresponding author: wtt31@126.com
} 
platform functional structure, etc. They provide significance reference for platform building, architecture and data transmission. However, there is no further research on the platform data mining and utilization, and there is no diagnostic function on vehicle exhaust and performance based on delivered data.

The key function of in-use vehicle remote monitoring platform is to diagnose vehicle failure status and monitor vehicle emission level. In fault diagnosis, OBD diagnostic method of vehicle itself has been fully developed, such as water temperature sensor fault diagnosis method of Fujitsu Ten Company ${ }^{[8]}$, based on temperature sensor, Cummins realizes fault diagnosis of internal combustion engine by monitoring the changing rate of intake temperature in a certain time interval ${ }^{[9]}$ and fault diagnosis of injection system by comparing the difference between theoretical urea injection and reduction in urea tank ${ }^{[10]}$; General Motors Company diagnoses system for over-injection or under-injection is based on the correlation between the control signal of the urea injection system nozzle and the fluctuation of its rail pressure ${ }^{[11]}$; As well as the fault diagnosis method of the dosing system based on the estimation of the urea injection volume model in the literature [12], the corresponding fault can be identified by comparing with the fixed calibration data inside the ECU under the normal condition of the vehicle sensor. But once the sensor signal is offset or the vehicle status have changed, it is difficult for these on-board diagnoses modular to identify tiny state anomalies which are frequently occurring only through local data of ECU. In terms of emission monitoring, China Automotive Technology Research Center Ltd. has achieved emission monitoring for heavy-duty vehicles through a remote emission management vehicle terminal and online monitoring platform ${ }^{[13]}$; Liu Shiguang ${ }^{[14]}$ analyzed the current situation of vehicle exhaust emission pollution based on OBD on-line monitoring. The above monitoring schemes focused on acquisition and calculation of emission information, which does not involve solutions for excessive vehicle emissions judgement, is difficult to adequately fulfill the requirements of platform users (regulatory authorities).

\section{Research objective}

\subsection{Exhaust and regulatory status of heavy duty diesel vehicles}

Heavy-duty diesels produce large amounts of nitrogen oxide $\left(\mathrm{NO}_{x}\right)$ and particulate matter (PM) emissions, so emission standards in various countries around the world, especially for heavy-duty vehicles, have been tightened in recent years. However, the emission of main pollutants from heavy-duty vehicles has not been effectively controlled in China. By the end of 2018, 18.8 million diesel trucks had been owned in China, accounting for $7.9 \%$ of 231 million vehicles. $\mathrm{NO}_{x}$ emissions from diesel trucks accounted for $60.3 \%$ of vehicle emissions and for particulate matter this number is $84.6 \%{ }^{[15]}$.

At present, CN_V emission regulations for heavy-duty (HD) diesels emission has been implemented nationwide, and in some regions, CN_VI emission regulations for HD diesels (CN_VI stage) has been implemented in advance for some special-purpose vehicles. However, on-road HD diesels are mainly consist of CN_IV \& CN_V vehicles. According to the actual emission results of heavy vehicles measured by various departments and research institutes ${ }^{[16]}$, the emission test results for the type-approved vehicles in the actual road through on-board emission equipment are much higher than the emission regulations. There are a lot of abnormal vehicle behaviors, such as vehicle emission failure without maintenance, NO $x$ control system cannot work, no consumption of urea, tampering by users or manufacturers, most of these behaviors are not effectively monitored.

\subsection{Cheating motivation and main forms of domestic heavy diesel vehicles}


Considering fuel economy, the post-treatment system of heavy duty diesel vehicles in $\mathrm{CN}_{-}$ IV and CN_V is mainly based on SCR technology..

As the urea solution required for SCR system operation will be continuously consumed in the normal use process and the supplementation of urea solution will bring corresponding economic investment, many commercial vehicle owners will reduce the consumption of urea solution and avoid the alarm of OBD system by various means during the use of SCR system. At present, common cheating methods include tampering with ECU software to turn off OBD function, pulling out temperature sensor completely, pulling out temperature sensor partially, using temperature sensor signal generator, NOx sensor partial dilution, pulling out NOx sensor, using NOx sensor signal generator, making urea injection flow back, etc.

\subsection{Remote monitoring of heavy duty diesel vehicles}

Under this background, the Central Committee of the CPC and the State Council clearly require the effective management of heavy-duty vehicles in use that meet the national emission standards of CN_III , CN_IV and CN_V in their "Opinions on Enhancing the Ecological Environment Protection in an All-round Way and Fighting the Strong Fighting Against Pollution" and the "Three-year Action Plan for Winning the Blue Sky Defense War" issued by the State Council. By installing remote emission management vehicle terminals(T$\mathrm{BOX}$ ) on in-use vehicles and based on the in-use vehicle remote monitoring platform, environmental protection regulatory agencies can real-time distinguish the vehicle emission status, in another hand, the OEM can monitor the vehicle operation status and handle vehicle problems in time ${ }^{[17]}$.

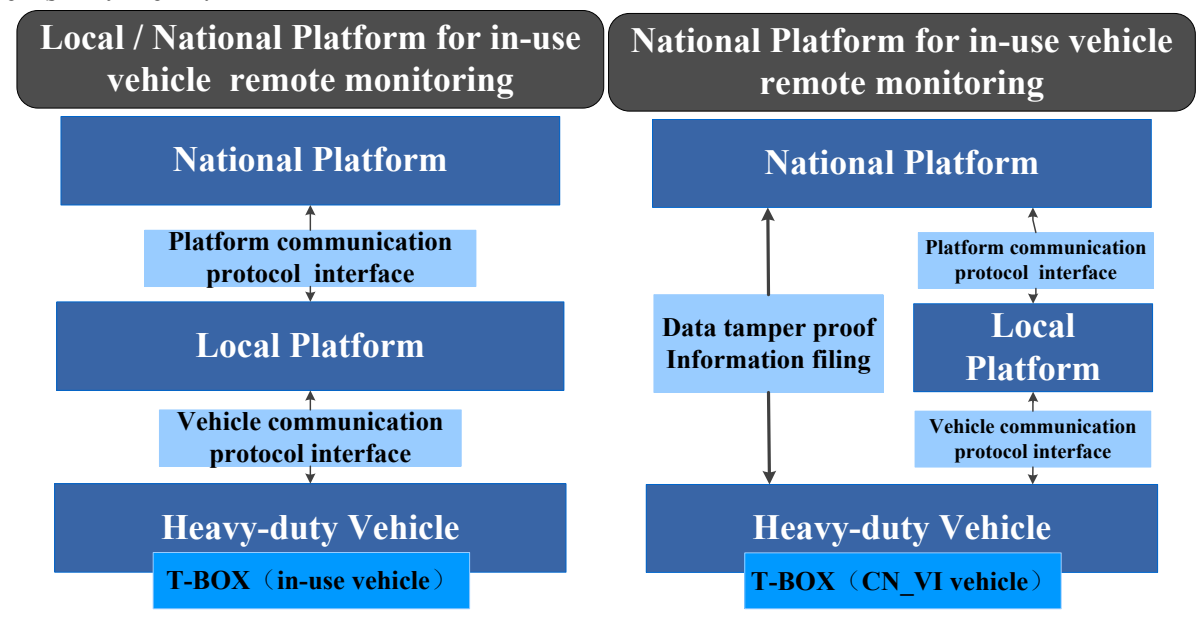

Fig. 1. Platform data interaction specification (in-use vehicle and CN_VI new vehicle).

The Ministry of Environmental Ecology is about to issue the Technical Specifications for Remote Emission Supervision System for Heavy Commercial Vehicles, which specifies the data communication interaction specifications between the on-board terminals of heavy duty vehicles used by the CN_VI new vehicle, the CN_IV vehicle and the CN_V vehicle and enterprise platforms, local platforms and national platforms, as shown in Fig. 1. The local platform is set up by the local ecological environment management department to collect, process, display and manage the data sent by the heavy vehicle remote emission management terminal in the $\mathrm{CN}_{-} \mathrm{V}$ and previous stages of the country, and to provide the heavy vehicle emission management services to the competent ecological environment departments. Referring to the design, construction and management standards of remote emission 
monitoring system for heavy vehicles, this paper establishes a standardized and multifunctional monitoring system by integrating on-board terminals, monitoring platforms and testing equipment, so as to effectively identify and solve the problems of excessive vehicle emissions.

\section{Vehicle remote supervision system}

\subsection{System composition}

The motor vehicle remote monitoring system is mainly composed of the vehicle remote monitoring terminal and the vehicle remote monitoring platform. The topology structure of the system is shown in Fig. 2. Vehicle terminal equipped with OBD diagnostic interface can collect the main operating parameters of the vehicle during operation. (e.g. engine speed, intake mass flow, fuel consumption, engine net output torque, SCR inlet temperature or SCR outlet temperature, urea injection volume, urea level sensor signal, downstream $\mathrm{NO}_{x}$ sensor measurement values, etc.) The platform has backstage services such as data storage, data cleaning, AI analysis, and front-end display functions such as vehicle information, driving and emission tracks, key area monitoring, fleet management, emission distribution, overrun warning, black-and-white list, etc. It can also interact and fuse data with remote sensing monitoring platform, vehicle annual inspection platform, road detection platform, etc. to provide comprehensive support for emission control.

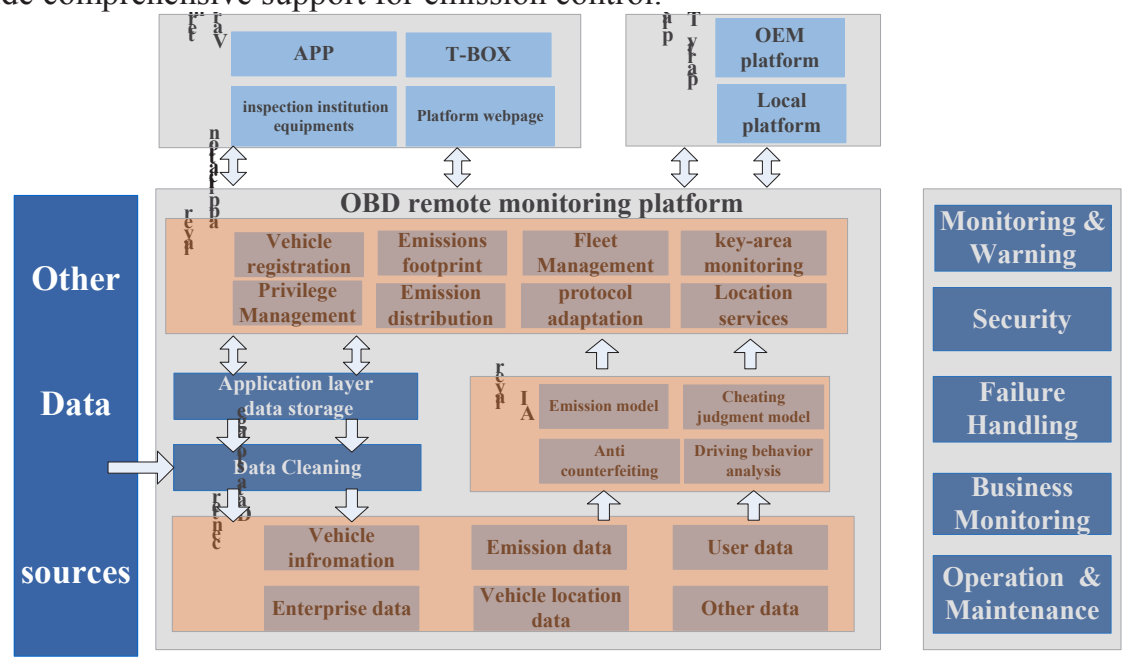

Fig. 2. Vehicle remote monitoring system.

\subsection{Working principle}

The working principle of the remote monitoring system for motor vehicles is illustrated in Fig. 3. OBD remote monitoring terminal (T-BOX) is usually installed in the vehicle OBD diagnostic interface, which can collect the main operating parameters of the vehicle during operation through the CAN bus (e.g. engine speed, intake mass flow, fuel consumption rate, engine net output torque, SCR inlet temperature/SCR outlet temperature, urea injection volume, urea level sensor signal, downstream $\mathrm{NO}_{x}$ sensor measurement etc.). Through the wireless communication module of the terminal, the data will be sent to the remote monitoring platform in the format specified by the platform using the $2 \mathrm{G} / 4 \mathrm{G}$ network. The 
platform can accept the data uploaded by the terminals installed on different vehicles, and carry out a series of data pre-processing, storage and calculation operations to achieve the normalized tracking of continuous, real-time and online status monitoring of vehicle operating condition information, I/M status information and pollutant emissions.

After processing a large number of long-term running data from on-line vehicles, many common cheating behavior of vehicles are identified based on temperature sensor signal diagnosis module, $\mathrm{NO}_{x}$ sensor signal diagnosis module, urea injection system working status diagnosis module etc. Based on the feedback results of the real state of the actual vehicle, train the neural network self-learning model that determines the diagnosis result, determine the threshold of core criteria and the weight factor of diagnosis result of sub-model. The learned model can accurately identify the abnormal using behavior of vehicles such as complete or partial pull-out of the temperature sensor, partial dilution of the $\mathrm{NO}_{x}$ sensor, pullout of the $\mathrm{NO}_{x}$ sensor, failure of the $\mathrm{NO}_{x}$ sensor, the using of temperature/ $\mathrm{NO}_{x}$ sensor signal generator, reflux of the injected urea, the using of poor quality urea and poor performance of SCR catalysts.

After confirming the rationality of the downstream $\mathrm{NO}_{x}$ sensor signal, the platform can pass the static registration information of the monitored vehicle (rated power, rated speed, maximum torque, engine displacement, emission standard, etc.), The power emission factor $(\mathrm{g} / \mathrm{kwh})$, mileage emission factor $(\mathrm{g} / \mathrm{km})$, and NOx emission concentration characteristics of a vehicle are calculated and extracted by combining the dynamic signals (vehicle speed, driving mileage, engine speed, coolant temperature, atmospheric pressure, engine torque, downstream $\mathrm{NO}_{x}$ sensor measurements, intake mass flow, fuel consumption rate, etc.) while the vehicle is running. The corresponding emission standards and operating conditions characteristics make a judgment of whether the vehicle's emission status exceeds the standard within a certain period of time, and make a blacklist and whitelist based on vehicles emission status.

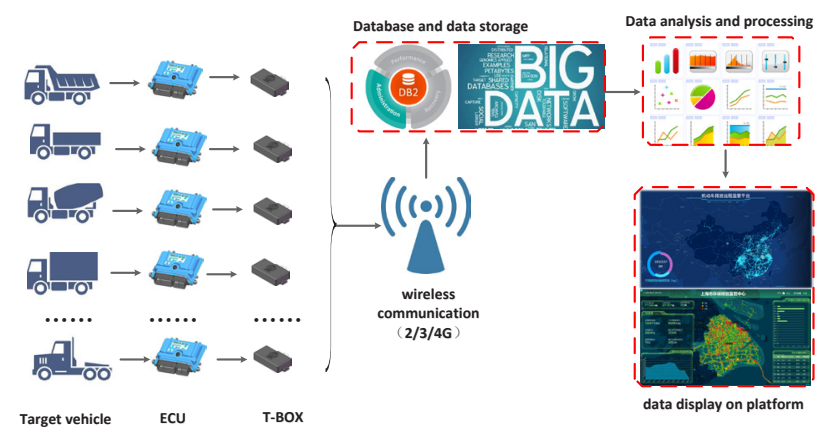

Fig. 3. On-road vehicle remote monitoring system.

\section{Cheating behavior diagnosis based on remote supervision platform}

\subsection{Unreasonable urea consumption}

Based on the correlation among the original exhaust data, tail-pipe exhaust data, urea injection system data and urea storage system data collected by the platform, the possible unreasonable urea consumption behavior is diagnosed remotely. Then, specific causes are judged by distinguishing strategy, so as to determine whether there are artificial malpractices during vehicle running. The specific monitoring logic is shown in Figure 4. 


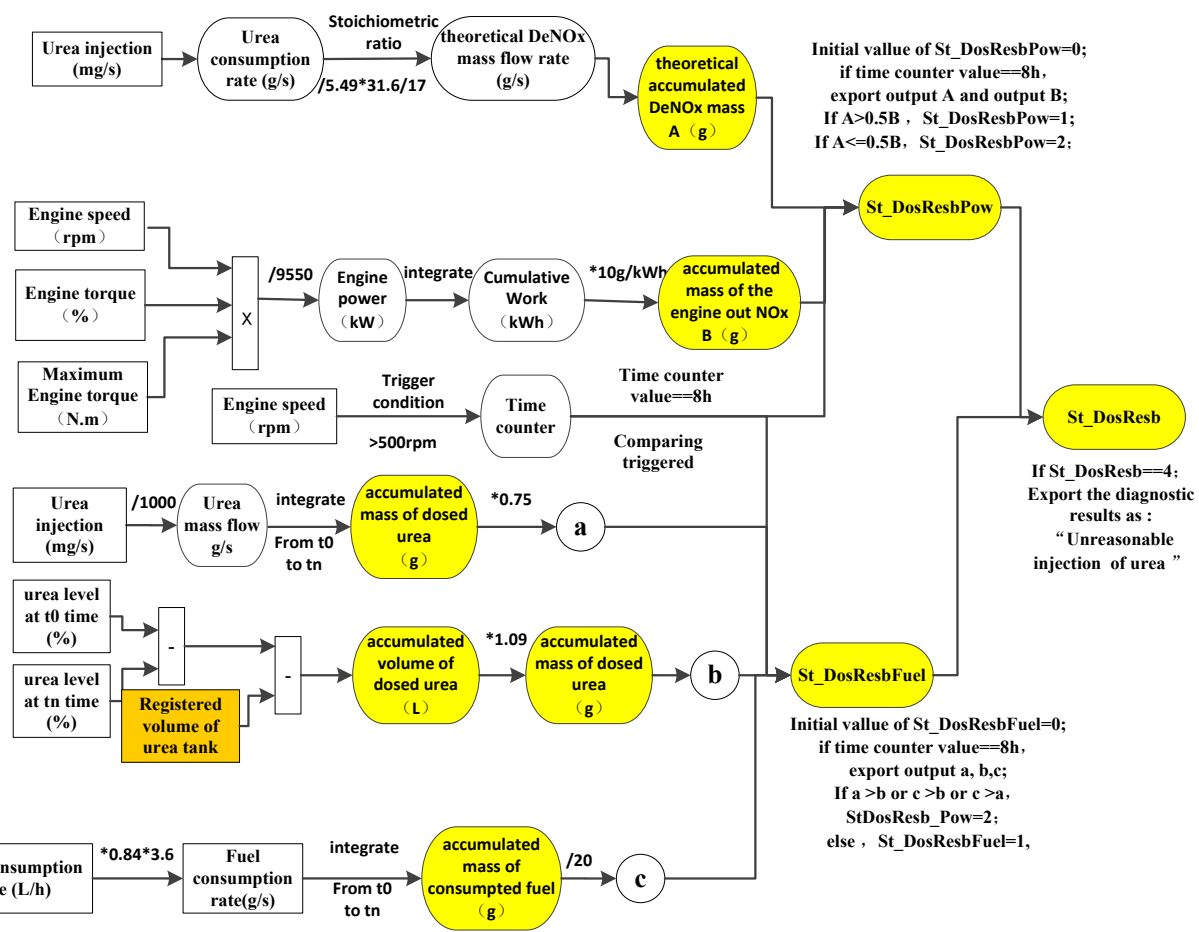

Fig. 4. Diagnostic method of urea consumption rationality.

The platform decides that urea consumption is too low in two ways. Method 1: By comparing the cumulative amount of urea consumed and the cumulative amount of fuel consumed over a relatively long period of time (e. $\mathrm{g}, 8 \mathrm{~h}$ engine running time), it can be obtained not only by integrating urea injection amount and fuel consumption rate into time, but also by long-term monitoring of urea filling action/state and fuel filling action/state through platform historical data. Urea consumption is considered to be too low when the ratio of cumulative fuel consumption to cumulative urea consumption exceeds a certain limit.

Method 2: The rationality of urea consumption is judged by calculating the cumulative engine work and cumulative urea consumption over a long period of time (e. g, $8 \mathrm{~h}$ engine running time). When the ratio of cumulative engine work and cumulative urea consumption exceeds a certain limit, it is considered that urea consumption is too low. The threshold is identified by the corresponding static parameter of target vehicle.

When the results are determined by both methods, the rationality of urea consumption diagnostic module finally outputs the diagnostic result (unreasonable urea consumption or not). The characteristic parameters of modules such as the thresholds are calibrated by engine and ATS operation data of from real road tests or platform historical data. 


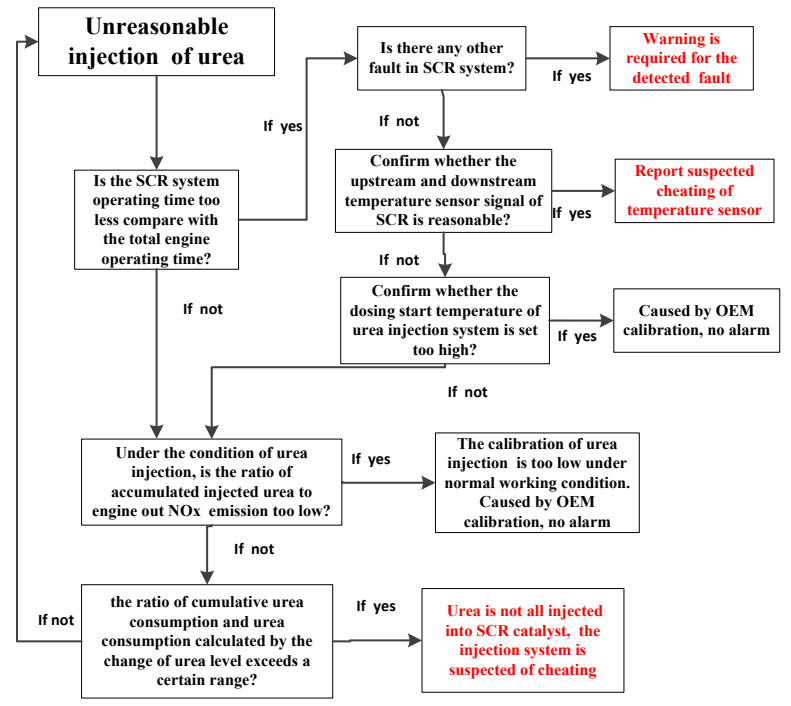

Fig. 5. Strategies for discriminating causes of unreasonable urea consumption.

The specific cause of unreasonable urea consumption is discriminated by the strategy shown in Fig. 5. The specific identification strategies are as follows: Firstly, after confirming the unreasonable consumption of urea, the diagnostic module will further calculate the proportion of SCR system working time to total engine running time. If this ratio is too low, further confirm whether the SCR system has a fault by reading the fault code, if there is a fault, report the relevant fault; if there is no fault, further confirm whether the upstream and downstream temperature sensor signal of SCR is reasonable. If the diagnosis result of temperature sensor signal is unreasonable, the suspected cheating of the temperature sensor is reported. Otherwise, it is necessary to further confirm whether the dosing-start temperature of urea in SCR system is set too high. If this temperature is too high, it should be identified but not alarmed (because is caused by OEM calibration).

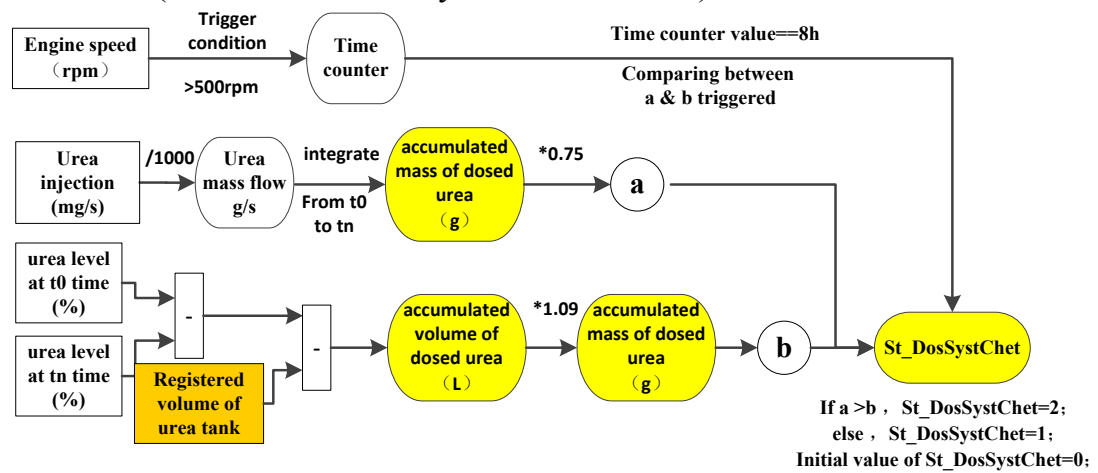

Fig. 6. Diagnostic method of suspected cheating in urea injection system.

Finally, confirming the proportionality relationship between the accumulated injected urea over a period of time when dosing on and the accumulated tail pipe $\mathrm{NO}_{x}$ emission. If this ratio is too low, it should be identified but not alarmed either (also caused by OEM calibration), If the ratio of urea consumption to tail pipe $\mathrm{NO}_{x}$ emission is within a reasonable range, but the DeNOx efficiency calculated by $\mathrm{NO}_{x}$ sensor downstream is too low, the corresponding relationship between the cumulative urea injection volume and urea level change in tank will be further compared. If the injection amount of urea is obviously higher 
than the level change of urea tank in a period of time, it is suspected that urea is not all injected into the SCR catalyst or there is a phenomenon of "exaggerate injection", as shown in Fig. 6. A diagnosis of "suspected cheating of the injection system" was made.

The above methods can detect unreasonable urea consumption, and can further identify a series of root causes that may lead to this unreasonable phenomenon, so that the abnormal working status of SCR system can be identified, and the inefficient SCR system can be precisely distinguished by environmental protection department, finally contributing to the reduction of $\mathrm{NO}_{x}$ emissions.

\subsection{Exhaust temperature sensor signal diagnosis}

Based on the exhaust temperature distribution characteristics under different torque conditions, an exhaust temperature sensor diagnosis method is proposed. Its main working principle is: first, the speed and torque segments are divided according to the power characteristics of the target vehicle. After the coolant temperature $>70{ }^{\circ} \mathrm{C}$, based on the measured value of exhaust temperature sensors at fixed position after working for a long time in the same speed/torque section of same vehicle, a reasonable temperature range of a certain speed/torque sections is calibrated by filtering and statistical methods. When the target diagnostic vehicle is working in this speed/torque period for more than a predetermined time, the rationality of temperature sensor signal is judged based on the diagnostic algorithm.

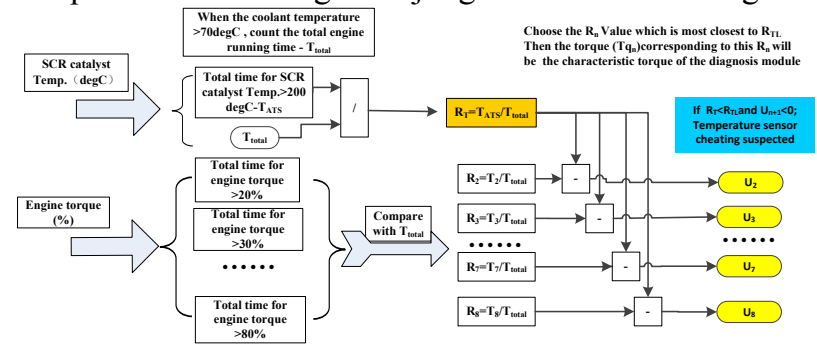

Fig. 7. Exhaust temperature sensor diagnosis method.

The diagnostic process is shown in Fig. 7, which mainly includes the following steps: (1) Collecting relevant parameters of target vehicle through T-BOX and uploading them to platform; (2) The platform configures the parameters of the diagnostic model as the characteristic parameters (including exhaust temperature time standard ratio - $\mathrm{R}_{\mathrm{TL}}$ and diagnostic characteristic torque $-\mathrm{T}_{\mathrm{n}}$ ) of such vehicles that already exist in the database; (3) After the engine running time exceeds the calibration limit, the diagnostic module counts the time that exhaust temperature exceed the calibrated temperature and the ratio $\left(\mathrm{R}_{\mathrm{T}}\right)$ of this time to total running time; (4) Comparing $\mathrm{R}_{\mathrm{T}}$ with $\mathrm{R}_{\mathrm{TL}}$; (5) For different torque segments, different time ratios of $R_{n}$ will be generated, values; (6) compared $R_{n+1}$ value with $R_{T L}$, If $R_{T}$ is less than $R_{n+1}$ and $R_{T}$ is less than $R_{T L}$, the unreasonable result of exhaust temperature sensor signal is obtained.

The calibration method for characteristic torque used for exhaust temperature sensor diagnostic module is shown in Fig. 8. Firstly, a normal vehicle with normal exhaust temperature sensor is selected for data acquisition, and the percentage of engine output torque, fuel consumption rate, exhaust temperature, engine speed, coolant temperature and other data are collected through T-BOX. Monitor coolant temperature when engine on. Once exceed $70{ }^{\circ} \mathrm{C}$, the time counter starts to counting the total running time. 


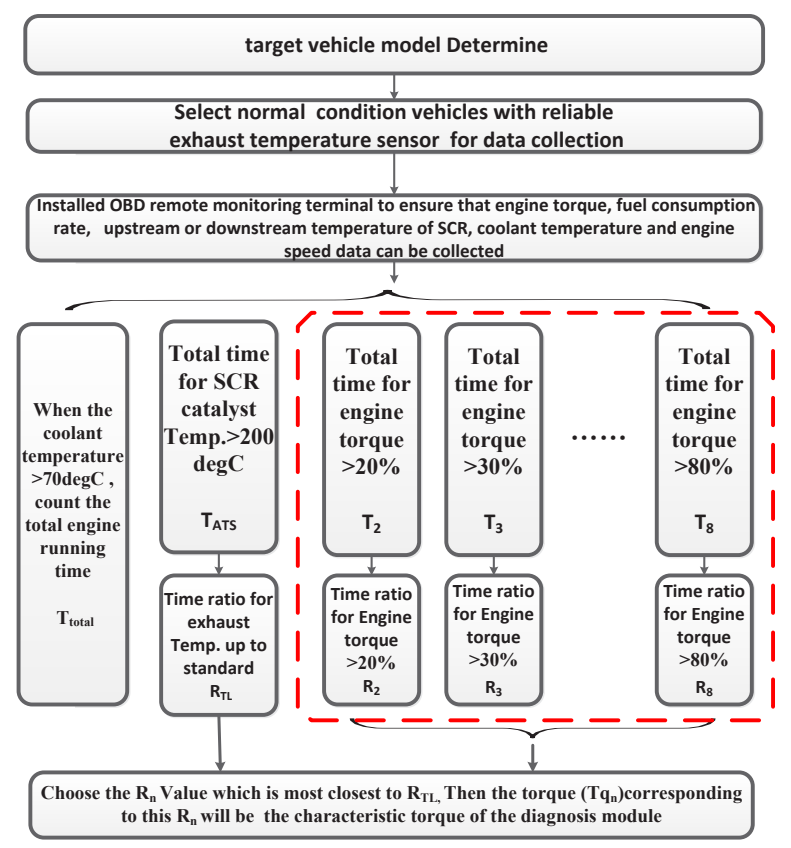

Fig. 8. characteristic torque calibration method.

After a long period of data collection, the time of engine torque $>20 \%,>30 \%,>40 \%,>$ $50 \%,>60 \%,>70 \%,>80 \%$ after engine heat-up was counted separately, the corresponding time to total running time is recorded as $\mathrm{R}_{2}, \mathrm{R}_{3}, \mathrm{R}_{4}, \mathrm{R}_{5}, \mathrm{R}_{6}, \mathrm{R}_{7}, \mathrm{R}_{8}$, respectively.

At the same time, counting the time that exhaust temperature sensor value is greater than the calibrated temperature (e.g. $200{ }^{\circ} \mathrm{C}$ ). The ratio of this time to the total running time is defined as the exhaust temperature up to standard time ratio $\left(\mathrm{R}_{\mathrm{T}}\right)$ and the value of this ratio is recorded. Selecting the torque that closest to $\mathrm{R}_{\mathrm{TL}}$ as the characteristic torque of the module.

\section{3 $\mathrm{NO}_{x}$ sensor signal diagnosis}

The platform judges the rationality of the $\mathrm{NO}_{x}$ sensor signal by using a model with selflearning function, and then determines whether the target vehicle has $\mathrm{NO}_{x}$ sensor problem. The diagnostic of $\mathrm{NO}_{x}$ sensor rationality in platform can be achieved through three solutions below:

1. As shown in Fig. 9, $\mathrm{NO}_{x}$ emission characteristics downstream of SCR increase with the increase of fuel injection or torque when SCR system has not ready for urea injection(always happened during engine cold start phase ), the peak and mean values of $\mathrm{NO}_{x}$ sensors during this period should be significantly higher than which urea have been injected. If the $\mathrm{NO}_{x}$ sensor value under this condition is continuously below the calibration limit, $\mathrm{NO}_{x}$ sensor cheating should be considered. The cold start discriminate module is shown in Fig. 10.

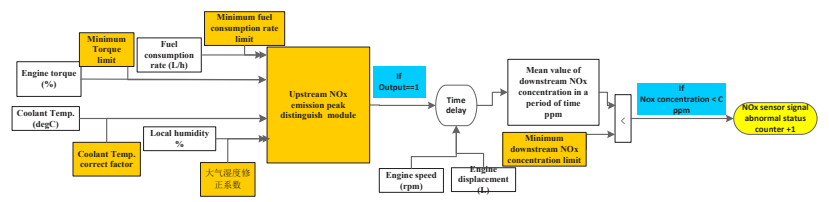

Fig. 9. NOx sensor diagnosis method 1. 


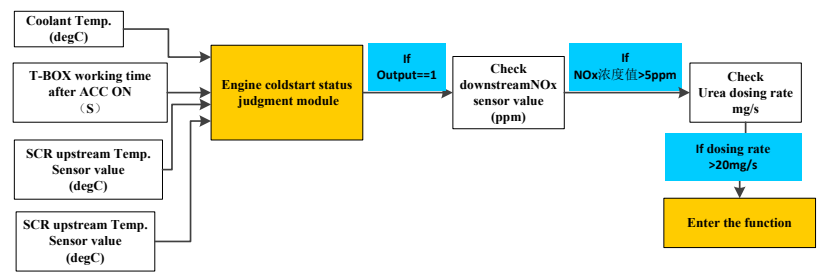

Fig. 10. Cold start discriminate module.

2. When the SCR system works, as long as the conversion efficiency is not $100 \%$ ( this is the normal status of V-based SCR system), the upstream $\mathrm{NO}_{x}$ emission change will be reflected in the downstream NOx emission change in a certain proportion. Based on this principle, considering the relationship between DeNOx efficiency and catalyst temperature in $\mathrm{CN}_{-} \mathrm{IV} / \mathrm{CN} \_\mathrm{V}$ SCR system, $\mathrm{NO}_{x}$ sensor signal authenticity is checked through $\mathrm{NO}_{x}$ sensor downstream value, SCR temperature and fuel injection /torque ${ }^{[18]}$.

3. According to tailpipe emission characteristics of $\mathrm{CN}_{-} \mathrm{IV} / \mathrm{CN}_{-} \mathrm{V}$ vehicles, the ratio of time measured by NOx sensor greater than a specific value (e.g. $50 \mathrm{ppm}$ ) to total working time is calculated. If this ratio is lower than a specific value (e.g. 10\%, which ultimately needs to be confirmed by a neural network self-learning model based on historical data), the sensor feedback value is considered to be significantly lower than the true emission level, and the working principle of this diagnostic method is shown in Figure 11.

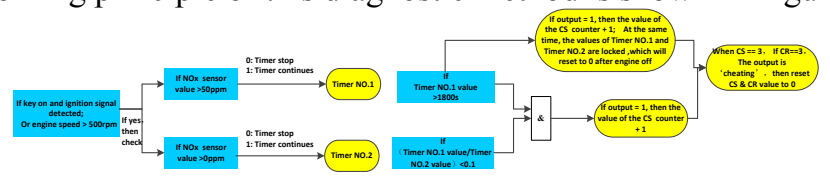

Fig. 11. NOx sensor signal diagnosis method 3 .

The accuracy of the above three judgment results is verified by real vehicles, and each algorithm is scored to establish a credible measurement table. Reasonable weight factors are allocated to the reliability of different types of vehicles when using different methods for judgment. Finally, the judgment results of the above three judgment methods are combined to make a final diagnostic results.

\section{Emission level confirming based on remote supervision platform}

The platform mainly calculates the $\mathrm{NO}_{x}$ emission by two methods, based on the results of both, it makes a comprehensive judgment with the status of vehicle emission level. The specific methods are as follows.

\subsection{Emission level confirming by $\mathrm{NO}_{x}$ emission factor and average concentration}




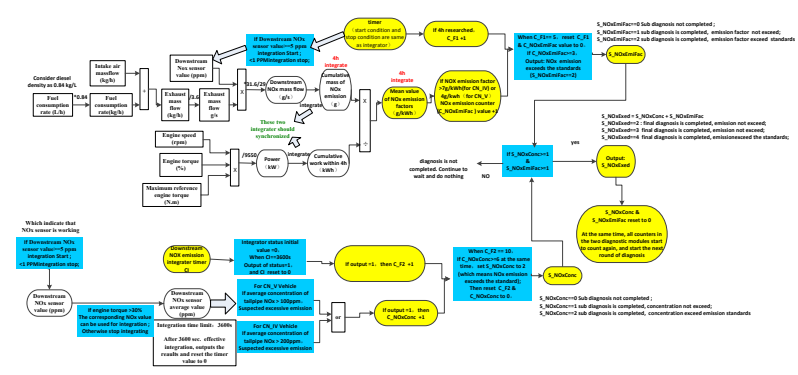

Fig. 12. Emission level confirming by NOx emission factor and average concentration.

When the rationality of the downstream NOx sensor signal is confirmed, The power emission factor $(\mathrm{g} / \mathrm{kwh})$, mileage emission factor $(\mathrm{g} / \mathrm{km})$, and $\mathrm{NO}_{x}$ emission concentration of the vehicle are calculated by using the registration information of the monitored vehicle (rated power, rated speed, maximum torque, engine displacement, emission standard, vehicle using, etc.) and the vehicle uploading data (vehicle speed, mileage, engine speed, coolant temperature, atmospheric pressure, engine torque, downstream $\mathrm{NO}_{x}$ sensor value, intake air mass flow, fuel consumption, etc.) when it is running. Finally output the result whether the emission level of this vehicle exceeds the corresponding standard within a certain period of time.

\subsection{Emission level confirming by PEMS-like $\mathrm{NO}_{x}$ emission factor}

Taking vehicle speed as input, different memory (urban road memory, suburban road memory, high-speed road memory) is established according to the PEMS test standard (GB17691-2018) to store the global data of certain working points under the corresponding vehicle speed conditions. Engine displacement is used to determine the total time to complete a PEMS-like $\mathrm{NO}_{x}$ emission calculation because the completion conditions of standard PEMS test are 4 7 WHTC cycles of work is done. The smaller the displacement, the longer the PEMS-like $\mathrm{NO}_{x}$ emission calculation. By confirming the vehicle type according to the vehicle registration information, the integral time limit (data amount) of urban, suburban and highway are determined, as shown in Fig. 13. 


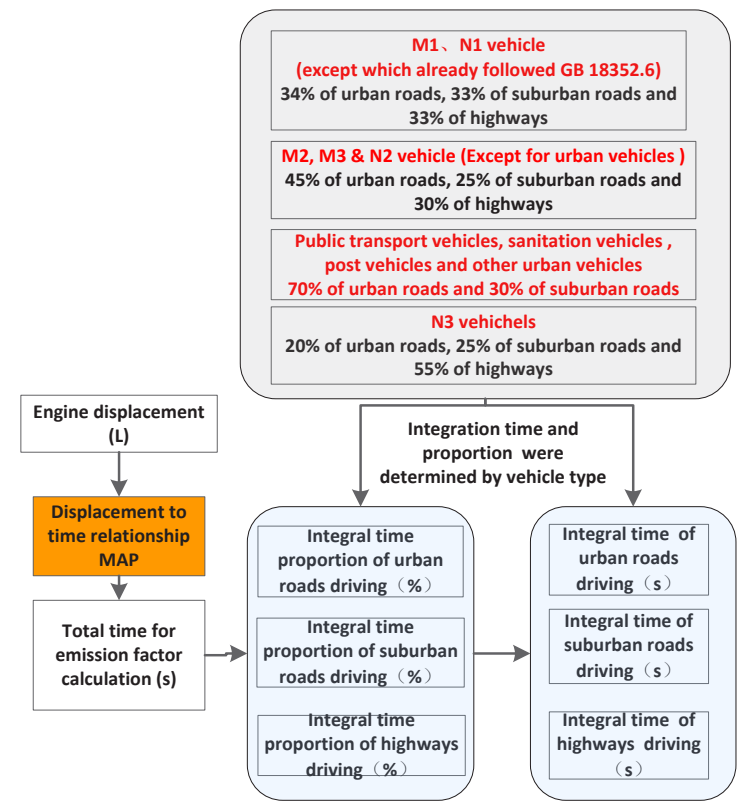

Fig. 13. Method for confirming integral time of PEMS-like cycle emission factor.

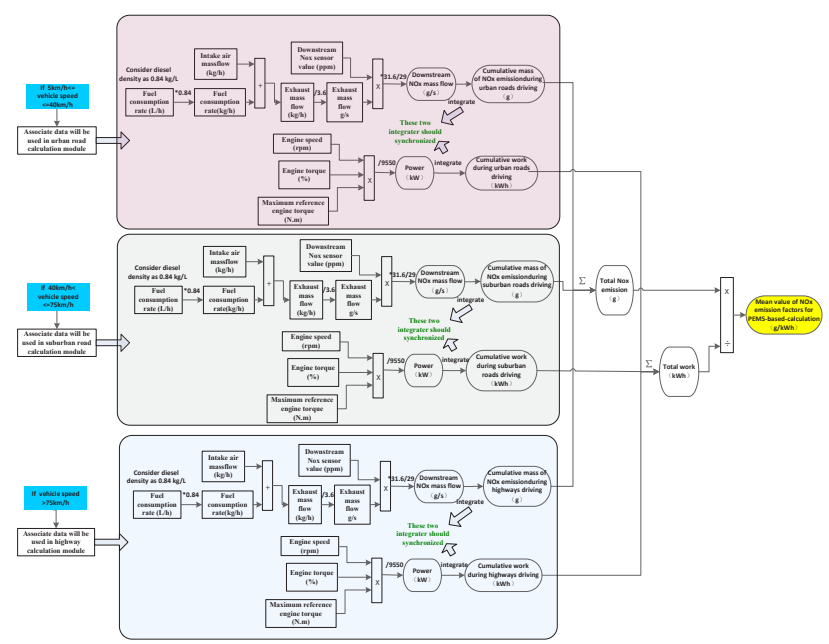

Fig. 14. calculation method of PEMS-like cycle emission factor.

When the integral time reaches the time limit of a modular, data storage stopped and the module outputs the corresponding $\mathrm{NO}_{x}$ emission quality and accumulated work. After the cumulative operating time of all modules reach each time limit, the $\mathrm{NO}_{x}$ emission quality and cumulative work of the three modules are summed and the final emission factor is calculated. Finally output the result whether the emission level of this vehicle exceeds the corresponding standard within a certain period of time by considering the operating conditions characteristics of the target vehicle.

\section{Summary}


For CN_IV and CN_V diesels, because the OBD system can only compare the running data of the vehicle itself with the corresponding diagnostic threshold calibrated in ECU, it is difficult to effectively identify the cheating behavior as mentioned above. In one hand, if the calibration threshold is too harsh, OEM will not accept this calibration dataset because the risk of misreport is high; in another hand, the loose diagnostic calibration threshold will lead to the failure or over-emission can only be reported under the extreme conditions, the overemission vehicle cannot be effectively monitored in this condition. What's more, ECU datasets without torque limit or OBD fault code also can be found in illegal market.

The emission characteristics, ATS operation characteristics and urea consumption characteristics of vehicle with cheating behavior are different from normal vehicles. Although the above cheating behaviors can hardly identified through OBD system and the running data of other vehicles cannot be used for comparing by single vehicle, the difference can be identified and the diagnosis can be finished with the help of the big data in platform.

This technical scheme is applicable to vehicles with T-BOX installed. This platform can accurately identify whether the vehicle has suspected cheating behavior and give out an emission results for emission supervision department through the modules by using the uploading data, which can reduce the cost of supervision and governance, improve the effectiveness of regulation and provide basic data for policy making.

\section{References}

1. Ding Jinquan, Liu Yingji. Remote vehicle performance monitoring system based on OBD, GPS and 3G Technology [J]. Transportation Energy Saving and Environmental Protection, 2011(03):55-58.

2. Qiu Yuan. Study on Vehicle Auto Real-Time Monitoring Terminal and Management System[D]. 2015. Guangxi University

3. Wang Yu, Hu Jiwen. Design of vehicle's long-distance management system based on OBD, GPS and 3G [J]. Journal of Huanggang Normal University, 2011, 31(6):67-68.

4. Liao Yanhui. Vehicle Remote Monitoring System Based on OBD2 [J]. AUTO TIME, 2018, No.295(04):29-30.

5. Yan Xupu. Design of Remote Monitoring System for Vehicle Tail Gas Emission Based on OBD Detection Data [D]. Beijing University of Technology. 2016

6. Liu Shiguang. Analysis of The Status Quo of Vehicle Emissions Pollution Based on OBD Online Monitoring [J]. Environment and development, 2019(5):181-181.

7. Dong Xiaoling. Remote Diagnosis and Monitor of Vehicle Based On OBD Device. [D]. Beijing Institute of Technology. 2016

8. Fujitsu Ten Limited. Fault diagnostic apparatus.US11363183,2008

9. CUMMINS ENGINE COMPANY INC.A system and method for detecting a fault condition in aninternal combustion engine, GB0306469. 20030423)

10. Jim Nebergall, Eric Hagen and Justin Owen.Selective Catalytic Reduction OnBoard Diagnostics: Past and Future Challenges.SAE Paper: 2005-01-3603

11. Yu Sun, Yue-Yun Wang, Chenfang Chang and Stephen. Detection of Urea Injection System Faults for SCR Systems.SAE Paper:2012-01-0431

12. Yue-Yun Wang, Yu Sun, Chen-Fang Chang, et al. Model-Based Fault Detection and Fault Tolerant Control of SCR Urea Injection System [J]. 2016, 65(6):4645-4654. 
13. Sun Yilong, Guo Yong, Wang Changyuan. Research on Data Consistency of Remote Emission Management Vehicle Terminals for Heavy-duty Vehicles [J] Small Internal Combustion Engine and Vehicle Technology. 2019, 48(02):7-12.

14. Liu Shiguang. Analysis of The Status Quo of Vehicle Emissions Pollution Based on OBD Online Monitoring [J]. Environment and development, 2019(5):181-181.

15. Ministry of Ecological Environment of the People's Republic of China. China Mobile Source Environmental Management Annual Report [M]; 2019

16. Li Mengliang, Li Jingyuan, Xu Yueyun. Study on in-use vehicle emission and its influencing factors [C]. Thesis Collection of the 14th Annual Meeting of Fuel and Lubrication Subcommittee of China Automotive Engineering Society. 2010

17. Department of Ecological Environment. GB-17691-2018 Limits and measurement methods for emissions from diesel fueled heavy-duty vehicles(CHINA VI)[S];2018-0622

18. Wang Y Y, Sun Y, Chang C F, et al. Model-based fault detection and fault-tolerant control of SCR urea in-jection systems[J]. IEEE Transactions on VehicularTechnology,2016,65(6):4645-4654. 